\title{
Xeno-free derivation and culture of human embryonic stem cells: current status, problems and challenges
}

Ting Lei ${ }^{1}$, Sandrine Jacob ${ }^{2}$, Imen Ajil-Zaraa ${ }^{1}$, Jean-Bernard Dubuisson ${ }^{2}$, Olivier Irion ${ }^{2}$, Marisa Jaconi ${ }^{3}$, Anis Feki ${ }^{1,2}$

${ }^{1}$ Laboratory of Stem Cell Research, Department of Obstetrics and Gynecology, Geneva University Hospitals, Geneva, Switzerland; ${ }^{2}$ Department of Obstetrics and Gynecology, Geneva University Hospitals, Geneva, Switzerland; ${ }^{3}$ Department of Pathology and Immunology, Faculty of Medicine, Geneva University, Geneva, Switzerland

Human embryonic stem cells (hESC) not only hold great promise for the treatment of degenerative diseases but also provide a valuable tool for developmental studies. However, the clinical applications of hESC are at present limited by xeno-contamination during the in vitro derivation and propagation of these cells. In this review, we summarize the current methodologies for the derivation and the propagation of hESC in conditions that will eventually enable the generation of clinical-grade cells for future therapeutic applications.

Keywords: embryonic stem cells, inner cell mass, derivation, feeder cells, xeno-free, differentiation

Cell Research (2007) 17:682-688. doi: 10.1038/cr.2007.61; published online 31 July 2007

\section{Introduction}

Since the first publication in 1998 [1], human embryonic stem cells (hESC) have attracted significant attention due to their dual ability to self-renew and to differentiate into all cell types of the body. This makes them excellent candidates for cell- and tissue-replacement therapies, as well as for the basic scientific research on human embryogenesis and diseases [2]. However, most hESC lines available to date have been directly or indirectly exposed to animal material during their derivation and/or propagation in vitro $[3,4]$. Although there are efforts to use the currently available hESC lines for clinical trials, such xeno-contaminated cells are generally judged unsuitable for transplantation because of the risk of zoonosis transmitted by animal pathogens and the potential activation of animal retroviruses, not to mention the possibility of immune rejection [5]. For therapeutic purposes, all steps for the production of hESC must avoid the use of animal components, i.e., culture systems using animal-free derivation methods, animal-free culture media,

Correspondence: Anis Feki

Tel: +1-41-22-38-24-392; Fax: +1-41-22-38-24-310

E-mail: anis.feki@hcuge.ch and animal-free substrates should be established to create and propagate clinical-grade hESC lines.

\section{Derivation of hESC}

hESC lines are conventionally derived from the inner cell mass (ICM) of pre-implantation stage blastocysts, of both good and poor quality [1, 6-8], which have been donated for research and would otherwise be discarded. Morula-stage embryos [9] or late-stage blastocysts (7-8 days) [10] may also be used to create hESC lines. Although all the hESC lines derived worldwide share the expression of characteristic pluripotency markers [11, 12], many differences are emerging between lines that may be more associated with the wide range of culture conditions in current use than with the inherent genetic variations of the embryos from which hESC were derived [13]. The reported success rate for hESC derivation is highly variable, possibly depending on the embryo quality and the culture protocol used. Very recently, Klimanskaya et al. published a new method for the derivation of hESC lines from single blastomeres obtained by a procedure similar to the one used for preimplantation genetic diagnosis, which could, theoretically, avoid embryo destruction in the future [14]. Alternatively, hESC lines were also successfully ob- 
tained from arrested embryos which stopped cleavage and failed to develop to morula and blastocysts in vitro [15]. Such possibilities are expected to partially ease the ethical concerns surrounding the conventional hESC derivation techniques, which destroy viable human embryos. Further studies are needed to validate the efficacy of these new derivation protocols.

\section{Isolation of ICM: immunosurgical versus mechanical methods}

Isolation of the ICM from blastocysts is a very important step in the derivation of hESC lines. Although several reports demonstrated the derivation of hESC lines from intact blastocysts after removal of zona pellucida [16-18], the outgrowth of trophectoderm at an early stage might inhibit the expansion of ICM and further reduce the success rate of hESC derivation [19]. Nearly all reported hESC lines to date have been efficiently obtained from ICM isolated by immunosurgery [20], a procedure that removes the outer trophectoderm epithelial cell layer from the blastocyst using anti-human whole-serum antibodies and guinea pig complement. However, the possible xeno-contamination in antiserum may limit its clinical use. Mechanical isolation of ICM serves therefore as a better alternative for the derivation of clinical-grade hESC lines, avoiding the exposure of blastocysts to animal antibodies. Human ESC lines have been successfully derived from manually isolated ICM [21]. In our laboratory, laser-hatching technology is employed to mechanically isolate the ICM. Efficiently used in assisted reproductive medicine, laser beams can be precisely delivered to drill the trophectoderm and favor ICM expulsion. Compared with the manual microdissection, laser-hatching technology requires minimal micromanipulation skills and maximally avoids xenogenic contamination by reagents. In mice, ESC lines have been successfully established from the ICM isolated by laser hatching [22], and we are now adapting and optimizing such techniques to dissect ICM from human embryos in

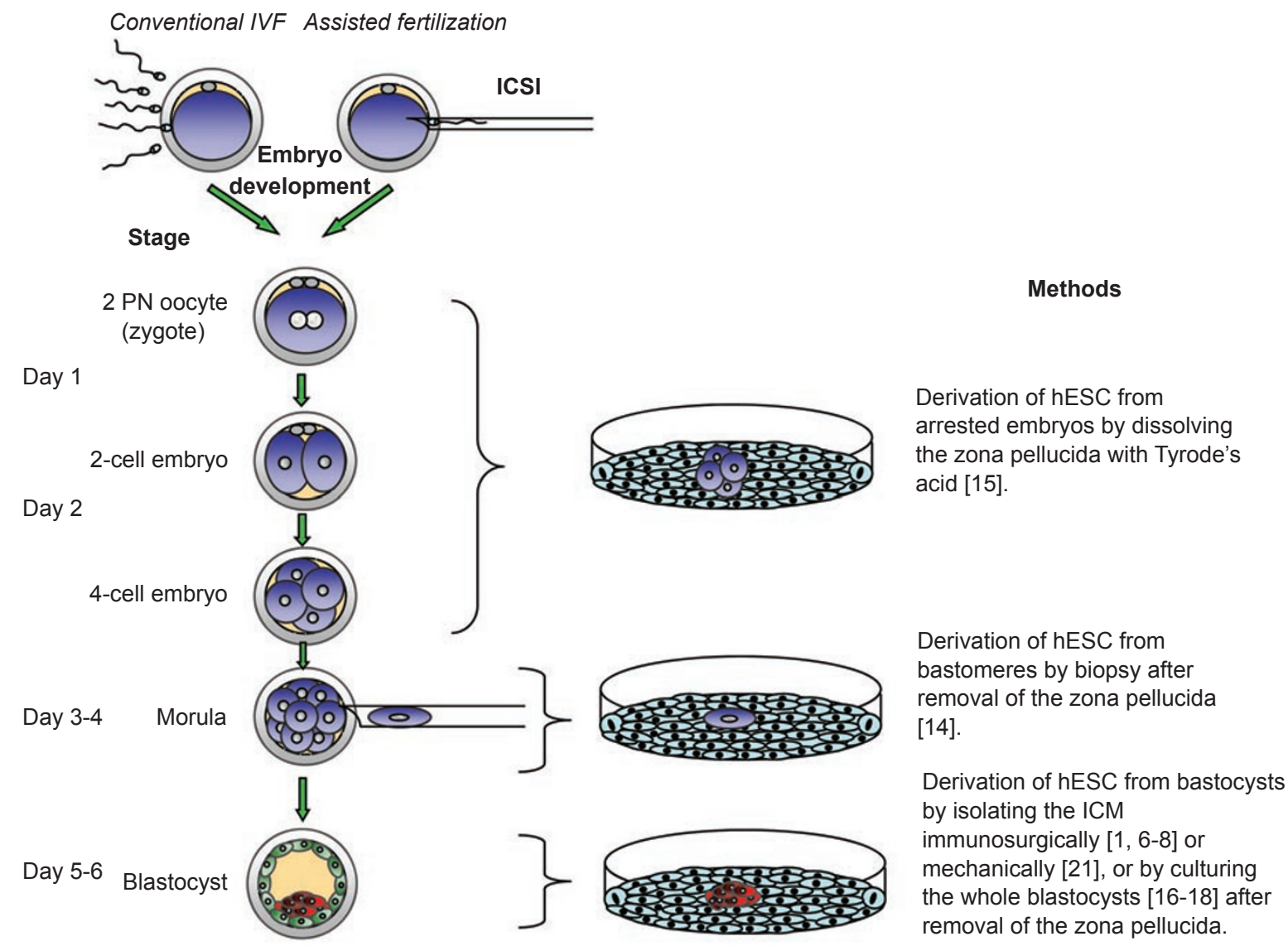

Figure 1 Derivation and culture methods for human embryonic stem cells. Here it is illustrated all embryo stages showed in the literature to give rise to human embryonic stem cell lines. IVF: In-vitro fertilization; ICSI: Intra-cytoplasmic sperm injection; PN: Pronucleus. 
animal-free conditions. Other methods to obtain intact and healthy ICM, including enzymatic digestion as employed for porcine blastocysts [23], should also be considered for human embryos if proven effective using recombinant enzymes.

\section{Feeder-dependent derivation of hESC}

To obtain ESC, the isolated ICM need to be placed on specific substrates and cultured in appropriate media (Figure 1). In most cases, ICM are cultured on mitotically inactivated feeder cells. Although irradiated or treated with mitomycin, feeder cells are still able to stimulate ESC growth and inhibit their differentiation through the secretion of specific growth factors and cytokines [24]. Like mouse ESC, hESC lines were firstly established on mouse embryonic fibroblasts (MEF) [1], in a medium containing fetal bovine serum (FBS). Such conditions are unsuitable for human cell transplantation since the exposure to animal components presents a serious risk of transmitting unidentified retroviruses and other pathogens to the patients. Although there is a report showing no evidence for hESC infection by animal feeder-derived viruses [25], concerns still remain over the clinical use of these cells. Furthermore, hESC cultured under xeno-contaminated conditions can present a non-human sialic acid, which is immunogenic to humans [5]. For therapeutic purposes and to eliminate, or at least to reduce, the xenogenic contamination from animal feeder cells and sera, human feeder cells and serum replacement (SR) have been recently employed for both culture and derivation of hESC lines [17, 26, 27]. One report also shows hESC lines established on feeder cells derived from hESC themselves [21]. However, none of these culture systems can be considered entirely animalfree since FBS was normally used to culture feeder cells, and SR is a reconstituted formulation still containing large amounts of bovine serum albumin as well as other proteins. In order to completely remove animal substances from the culture system, several teams have employed human serum in culture media $[16,28]$. hESC lines derived under such conditions are expected to meet the criteria for clinical applications.

\section{Feeder-free derivation of $h E S C$}

Like serum, feeder cells are a source of variability in experimental conditions and are also a concern for future transplantation of hESC derivatives to patients. A recent study successfully established a hESC line on the extracellular matrix (ECM) prepared from MEF [29]. Despite the immunosurgical ICM isolation and the culture in SRsupplemented medium, the use of such ECM represents a significant advance as, if proven efficient, ECM of human origin would avoid the above-mentioned pathogenic risks, as well as the possible interference of living feeder cells in future transplantation therapies. In addition, feeder cellderived ECM can be easily sterilized and stored for clinical applications. Another recent report [30] described the derivation of two hESC lines in a defined culture medium containing components solely derived from recombinant sources or purified from human material, on substrate formed by human ECM components. Although these culture conditions are laborious and costly, and may lead to abnormal karyotype during long-term culture, hESC lines derived and propagated in this xeno-free and feederindependent system would be more directly applicable to clinical use. Such culture conditions also provide a basis for further simplifying culture requirements in studies investigating the molecular mechanisms of hESC self-renewal and differentiation. We are presently testing the capability of human feeder cell-derived ECM to support prolonged undifferentiated growth of hESC, aiming at the establishment of clinical-grade lines in suitably defined xeno-free culture conditions.

\section{Clonal derivation of $h E S C$}

hESC derived from ICM of blastocysts should be considered as heterogenous [31]. Isolation of a homogenous pool of differentiated hESC is necessary for basic studies like drug development and toxicity testing. Moreover, for therapeutic applications, the removal of undifferentiated, potentially tumorigenic cells will also be a requirement, or at least malfunctioning cells should be removed using cell ablation strategies, before and/or after engraftment.

New hESC lines have been clonally derived from the existing ones [32-35]. However, unlike mouse ESC, the clonal efficiency of hESC is extremely low, as hESC are sensitive to single-cell disaggregation and recover poorly when plated at clonal density. Indeed, cell-cell interactions seem critical for efficient hESC propagation, since the loss of gap junctions between hESC can increase cell apoptosis and inhibit hESC colony growth [36]. For these reasons, $\mathrm{hESC}$ are routinely passaged in cell clumps, produced by mechanical cutting and/or mild enzymatic treatment $[1,8]$, which is an obstacle to large-scale propagation of hESC for clinical use. Nevertheless, clonal survival of hESC can be enhanced by culturing in physiologic oxygen $(2 \%)$ [37], or in the presence of neurotrophins [38]. Therefore, the efficiency of hESC derivation might be improved at low oxygen concentration and/or with neurotrophins in culture medium.

\section{Passaging of hESC: mechanical versus enzymatic methods}

When the feeder cells become old or the colonies are 
too dense or too large, hESC need to be split for continuing culture. Passaging of hESC falls into two categories - mechanical and enzymatic. Clearly, the passaging technique is critical for maintaining undifferentiated and karyotypically stable hESC lines. Mechanical passaging is performed by dissociating the colonies into small clumps through manually scraping and cutting and transferring them to new culture plates covered with feeder cells or appropriate substrates. This is labor-intensive and time-consuming, and it normally generates variable cluster size and results in inconsistent cell distribution. On the other hand, enzymatic passaging dissociates the hESC colonies using enzymes such as animal-derived collagenase, dispase, and trypsin [39], xeno-free recombinant enzyme [16] or enzyme-free cell dissociation buffer [40], allowing a relatively consistent and standardized passaging of hESC. In view of clinical applications, enzymatic passaging is advantageous since it enables large-scale expansion of hESC. However, emerging evidence suggests that excessive and/or frequent dissociation to single cells may lead to karyotype abnormalities [ 41 , 42]. In contrast, mechanical methods of passaging allow selective transfer of exclusively undifferentiated colonies and seem to better maintain genetic stability. Since the process of manual colony dissection limits the practical use of mechanical passaging in bulk hESC culture, many researchers combine both enzymatic and mechanical passaging methods for long-term hESC maintenance [8, 43]. Indeed, hESC lines initially derived and passaged by mechanical methods have been subsequently adapted to enzymatic passaging for practical reasons [6]. Automated mechanical passaging of hESC, which incorporates the advantages of mechanical dissection without sacrificing the practical benefits of enzymatic passaging, was also developed [44]. To overcome the low resistance of hESC to single-cell dissociation during passaging, recent studies have achieved the culture of newly derived hESC lines which keep stable karyotype in long-term culture by enzymatic bulk passage $[45,46]$. These single-cell dissociation-resistant hESC lines would be valuable for clinical applications. Nevertheless, hESC in long-term culture need to be routinely checked for their chromosomal stability.

\section{Propagation of hESC}

Considerable progress has been made towards the definition of xeno-free culture conditions allowing the propagation of undifferentiated hESC, thus significantly improving the clinical potential of these cells.

\section{Feeder-dependent propagation of $h E S C$}

To eliminate the xeno-contamination of mouse feeder cells, human feeder cells have been derived and shown to support the undifferentiated growth of hESC [28]. The existing hESC lines can maintain their pluripotency on several types of human feeder cells [47], or on the feeder cells derived from hESC themselves [21, 48]. However, most commonly used human feeders have been exposed to animal components during their isolation and culture [24], and the animal-derived SR is still needed to maintain hESC growth under these conditions, potentially making $h E S C$ xeno-contaminated and unsuitable for cell replacement therapies. In addition, hESC cultured in SR medium present immunogenic sialic acids on their surface; although the contamination with non-human sialic acids can be diluted (and probably lost) in hESC after long-term culture under xeno-free conditions, they may still represent a risk to transplantation [5]. In view of clinical applications, it is mandatory to establish clinical-grade feeder cell lines for hESC derivation and propagation, while animal serum and SR should be eliminated from culture media for both feeder cells and hESC. Most recently, Ellerstrom et al. [16] established xeno-free human foreskin fibroblast feeders for $\mathrm{hESC}$ derivation, in a medium supplemented with human serum and devoid of any animal material. The hESC lines derived and propagated in such systems are therefore advantageous for possible therapeutic purposes.

\section{Feeder-free propagation of hESC}

The necessity of feeder cells limits the capacity to meet the large-scale culture demands for clinical applications, as well as for genetic manipulation of hESC in basic research. Furthermore, establishment of clinical-grade feeder cell lines for hESC culture is costly. Xu et al. [49] first demonstrated a successful feeder-free hESC culture system in which undifferentiated cells can be maintained in the long term on a Matrigel layer in MEF-conditioned medium. Alternatively, hESC-derived fibroblasts can also be used to produce conditioned medium capable of supporting undifferentiated growth of hESC on Matrigel [48, 50]. However, Matrigel is a soluble basement membrane extract from mouse tumors containing several types of ECM and other unknown factors, and MEF-conditioned medium also contains undefined animal components, which limits its use for clinical-grade hESC culture. As a step forward, a single ECM component such as laminin or fibronectin, of both animal and human origin, has also been successfully used to support undifferentiated growth of hESC in either MEF-conditioned medium or medium supplemented with SR and various growth factors [49, 51, 52]. In these conditions the possible xeno-contamination in substrates is reduced, but in culture media it remains and a risk to future clinical applications may still persist.

In order to optimize the culture conditions, great efforts have been made in identifying conditioned media 
components essential for hESC self-renewal, as a crucial prerequisite for the eventual applications of $\mathrm{hESC}$ in the treatment of human diseases. High throughput screening methods have been employed to investigate the protein composition of media conditioned by feeder cells of either animal or human origin $[53,54]$, providing preliminary insight into the possible feeder cell-secreted factors that support the growth of hESC. Basic fibroblast growth factor (bFGF) appears to play a key role in sustaining hESC self-renewal, and is included in nearly all the reported medium formulations for $\mathrm{hESC}$ derivation and propagation. Indeed, certain existing hESC lines have been successfully maintained over a long term in unconditioned medium supplemented with a high concentration of bFGF [55]. Suppression of bone morphogenetic proteins (BMP) also seems to be important for hESC propagation. Noggin, a BMP antagonist, synergizes with bFGF in sustaining the proliferation of undifferentiated hESC in the absence of feeder cells and conditioned medium [56]. Although the possible xeno-contamination of MEF-conditioned medium is avoided in these culture systems, exogenous factors in unconditioned medium (e.g. SR) and in culture substrates (e.g. Matrigel) still pose a risk to therapeutic applications. To circumvent this issue, Stojkovic et al. [57] reported the growth of undifferentiated hESC on human serum-coated plates in SR-containing medium conditioned by human embryonic fibroblasts derived from hESC, which reduces the exposure of hESC to animal ingredients. Recently, several chemical-defined culture media, in which the SR is replaced with a cocktail of recombinant growth factors and cytokines, have been developed to sustain undifferentiated propagation of existing $\mathrm{hESC}$ lines on Matrigel or on human-derived ECM in short-term studies [58-60]. Such animal-free media should significantly facilitate the use of hESC in therapeutic applications. However, as discussed above, most available hESC cell lines are xeno-contaminated and their propagation in animal-free medium cannot completely eliminate the exogenous and immunogenic factors in culture [5]. For clinical purposes, it would require starting with newly derived hESC that have never been exposed to animal products. As a step forward, Ludwig et al. [30] successfully derived and propagated two new hESC lines in defined medium on human-derived substrates, although immunosurgery is still employed in their work to isolate the ICM. Most recently, Fletcher et al. [61] reported the first hESC line derived without direct exposure to any undefined animal products. We expect such approaches to facilitate the clinical applications of hESC and to provide a platform for further optimizing culture requirements for undifferentiated growth of hESC.

It should be noted that, compared with the culture on feeder cells, the feeder-free system is less optimal for sustaining the undifferentiated growth of hESC, as differentiation on the edges of the colonies can frequently be seen in these conditions. Furthermore, feeder-free cultures of hESC might result in chromosomal changes, such as gain of chromosomes 17q and 12 [62], which should be a concern for the future applications of these cells in transplantation therapies.

\section{Other concerns}

Despite the elimination of all animal components from the derivation, propagation and passaging process, there are still additional issues that should be addressed prior to the clinical applications of hESC. A general concern in all allogenic transplantations is the exposure of hESC to human feeder cells and human proteins in culture that may introduce unknown human pathogens into patients on cell transplantation therapy. Therefore, all the human substances used during the establishment and propagation of hESC must be extensively screened for the final therapeutic applications. Besides, as reviewed elsewhere [63], other roadblocks, such as tumorigenicity and genetic compatibility, also stand in the way of progress towards hESC-based cell therapies and these need to be removed before the clinical potential of hESC can be exploited.

\section{Conclusion}

To develop a clinical-grade $\mathrm{hESC}$ line, it is a prerequisite to show that all steps in the derivation, passaging and culturing of hESC are completely free of animal products. Ideally, such hESC lines should be derived from mechanically isolated ICM in a culture system which uses human-derived ECM and chemically defined medium, and which keeps normal karyotype for long term with xeno-free enzymatic passaging. Before the molecular mechanisms of hESC self-renewal are fully uncovered, it is currently acceptable to derive and culture hESC lines for possible therapeutic applications on human feeder cells in media supplemented with proteins that are solely of human origin.

\section{Acknowledgments}

We gratefully acknowledge the support of the Swiss National Foundation for Scientific Research.

\section{References}

1 Thomson JA, Itskovitz-Eldor J, Shapiro SS, et al. Embryonic stem cell lines derived from human blastocysts. Science 1998; 282:1145-1147.

2 Trounson A. The production and directed differentiation of human embryonic stem cells. Endocr Rev 2006; 27:208-219. 
3 Wang WH, Sun XF. Human embryonic stem cell lines are contaminated: what should we do? Hum Reprod 2005; 20:29872989.

4 Skottman H, Dilber MS, Hovatta O. The derivation of clinical-grade human embryonic stem cell lines. FEBS Lett 2006; 580:2875-2878.

5 Martin MJ, Muotri A, Gage F, Varki A. Human embryonic stem cells express an immunogenic nonhuman sialic acid. Nat Med 2005; 11:228-232.

6 Cowan CA, Klimanskaya I, McMahon J, et al. Derivation of embryonic stem-cell lines from human blastocysts. N Engl J Med 2004; 350:1353-1356.

7 Chen H, Qian K, Hu J, et al. The derivation of two additional human embryonic stem cell lines from day 3 embryos with low morphological scores. Hum Reprod 2005; 20:2201-2206.

8 Reubinoff BE, Pera MF, Fong CY, Trounson A, Bongso A. Embryonic stem cell lines from human blastocysts: somatic differentiation in vitro. Nat Biotechnol 2000; 18:399-404.

9 Strelchenko N, Verlinsky O, Kukharenko V, Verlinsky Y. Moruladerived human embryonic stem cells. Reprod Biomed Online 2004; 9:623-629.

10 Stojkovic M, Lako M, Stojkovic P, et al. Derivation of human embryonic stem cells from day-8 blastocysts recovered after three-step in vitro culture. Stem Cells 2004; 22:790-797.

11 Andrews PW, Benvenisty N, McKay R, et al. The International Stem Cell Initiative: toward benchmarks for human embryonic stem cell research. Nat Biotechnol 2005; 23:795-797.

12 Brivanlou AH, Gage FH, Jaenisch R, et al. Stem cells. Setting standards for human embryonic stem cells. Science 2003; 300:913-916.

13 Allegrucci C, Young LE. Differences between human embryonic stem cell lines. Hum Reprod Update 2007; 13:103-120.

14 Klimanskaya I, Chung Y, Becker S, Lu SJ, Lanza R. Human embryonic stem cell lines derived from single blastomeres. Nature 2006; 444:512.

15 Zhang X, Stojkovic P, Przyborski S, et al. Derivation of human embryonic stem cells from developing and arrested embryos. Stem Cells 2006; 24:2669-2676.

16 Ellerstrom C, Strehl R, Moya K, et al. Derivation of a xeno-free human embryonic stem cell line. Stem Cells 2006; 24:21702176.

17 Genbacev O, Krtolica A, Zdravkovic T, et al. Serum-free derivation of human embryonic stem cell lines on human placental fibroblast feeders. Fertil Steril 2005; 83:1517-1529.

18 Kim HS, Oh SK, Park YB, et al. Methods for derivation of human embryonic stem cells. Stem Cells 2005; 23:1228-1233.

19 Pickering SJ, Braude PR, Patel M, et al. Preimplantation genetic diagnosis as a novel source of embryos for stem cell research. Reprod Biomed Online 2003; 7:353-364.

20 Solter D, Knowles BB. Immunosurgery of mouse blastocyst. Proc Natl Acad Sci USA 1975; 72:5099-5102.

21 Wang Q, Fang ZF, Jin F, et al. Derivation and growing human embryonic stem cells on feeders derived from themselves. Stem Cells 2005; 23:1221-1227.

22 Tanaka N, Takeuchi T, Neri QV, Sills ES, Palermo GD. Laser-assisted blastocyst dissection and subsequent cultivation of embryonic stem cells in a serum/cell free culture system: applications and preliminary results in a murine model. J Transl Med 2006; $4: 20$.
23 Li M, Zhang D, Hou Y, et al. Isolation and culture of embryonic stem cells from porcine blastocysts. Mol Reprod Dev 2003; 65:429-434.

24 Stacey GN, Cobo F, Nieto A, et al. The development of 'feeder' cells for the preparation of clinical grade hES cell lines: challenges and solutions. Journal of Biotechnology 2006; 125:583588.

25 Amit M, Winkler ME, Menke S, et al. No evidence for infection of human embryonic stem cells by feeder cell-derived murine leukemia viruses. Stem Cells 2005; 23:761-771.

26 Lee JB, Lee JE, Park JH, et al. Establishment and maintenance of human embryonic stem cell lines on human feeder cells derived from uterine endometrium under serum-free condition. Biol Reprod 2005; 72:42-49.

27 Inzunza J, Gertow K, Stromberg MA, et al. Derivation of human embryonic stem cell lines in serum replacement medium using postnatal human fibroblasts as feeder cells. Stem Cells 2005; 23:544-549.

28 Richards M, Fong CY, Chan WK, Wong PC, Bongso A. Human feeders support prolonged undifferentiated growth of human inner cell masses and embryonic stem cells. Nat Biotechnol 2002; 20:933-936.

29 Klimanskaya I, Chung Y, Meisner L, et al. Human embryonic stem cells derived without feeder cells. Lancet 2005; 365:16361641.

30 Ludwig TE, Levenstein ME, Jones JM, et al. Derivation of human embryonic stem cells in defined conditions. Nat Biotechnol 2006; 24:185-187.

31 Stewart MH, Bosse M, Chadwick K, et al. Clonal isolation of hESCs reveals heterogeneity within the pluripotent stem cell compartment. Nat Methods 2006; 3:807-815.

32 Amit M, Carpenter MK, Inokuma MS, et al. Clonally derived human embryonic stem cell lines maintain pluripotency and proliferative potential for prolonged periods of culture. Dev Biol 2000; 227:271-278.

33 Heins N, Lindahl A, Karlsson U, et al. Clonal derivation and characterization of human embryonic stem cell lines. J Biotechnol 2006; 122:511-520.

34 Hewitt Z, Forsyth NR, Waterfall M, et al. Fluorescence-activated single cell sorting of human embryonic stem cells. Cloning Stem Cells 2006; 8:225-234.

35 Sidhu KS, Tuch BE. Derivation of three clones from human embryonic stem cell lines by FACS sorting and their characterization. Stem Cells Dev 2006; 15:61-69.

36 Wong RC, Dottori M, Koh KL, et al. Gap junctions modulate apoptosis and colony growth of human embryonic stem cells maintained in a serum-free system. Biochem Biophys Res Commun 2006; 344:181-188.

37 Forsyth NR, Musio A, Vezzoni P, et al. Physiologic oxygen enhances human embryonic stem cell clonal recovery and reduces chromosomal abnormalities. Cloning Stem Cells 2006; 8:16-23.

38 Pyle AD, Lock LF, Donovan PJ. Neurotrophins mediate human embryonic stem cell survival. Nat Biotechnol 2006; 24:344350 .

39 Hoffman LM, Carpenter MK. Characterization and culture of human embryonic stem cells. Nat Biotechnol 2005; 23:699-708.

40 Zeng X, Cai J, Chen J, et al. Dopaminergic differentiation of human embryonic stem cells. Stem Cells 2004; 22:925-940. 
41 Buzzard JJ, Gough NM, Crook JM, Colman A. Karyotype of human ES cells during extended culture. Nat Biotechnol 2004; 22:381-382.

42 Mitalipova MM, Rao RR, Hoyer DM, et al. Preserving the genetic integrity of human embryonic stem cells. Nat Biotechnol 2005; 23:19-20.

43 Oh SK, Kim HS, Park YB, et al. Methods for expansion of human embryonic stem cells. Stem Cells 2005; 23:605-609.

44 Joannides A, Fiore-Heriche C, Westmore K, et al. Automated mechanical passaging: a novel and efficient method for human embryonic stem cell expansion. Stem Cells 2006; 24:230-235.

45 Suemori H, Yasuchika K, Hasegawa K, et al. Efficient establishment of human embryonic stem cell lines and long-term maintenance with stable karyotype by enzymatic bulk passage. Biochem Biophys Res Commun 2006; 345:926-932.

46 Hasegawa K, Fujioka T, Nakamura Y, Nakatsuji N, Suemori H. A method for the selection of human embryonic stem cell sub-lines with high replating efficiency after single cell dissociation. Stem Cells 2006; 24:2649-2660.

47 Richards M, Tan S, Fong CY, et al. Comparative evaluation of various human feeders for prolonged undifferentiated growth of human embryonic stem cells. Stem Cells 2003; 21:546-556.

48 Stojkovic P, Lako M, Stewart R, et al. An autogeneic feeder cell system that efficiently supports growth of undifferentiated human embryonic stem cells. Stem Cells 2005; 23:306-314.

$49 \mathrm{Xu} \mathrm{C}$, Inokuma MS, Denham J, et al. Feeder-free growth of undifferentiated human embryonic stem cells. Nat Biotechnol 2001; 19:971-974.

$50 \mathrm{Xu}$ C, Jiang J, Sottile V, et al. Immortalized fibroblast-like cells derived from human embryonic stem cells support undifferentiated cell growth. Stem Cells 2004; 22:972-980.

51 Amit M, Shariki C, Margulets V, Itskovitz-Eldor J. Feeder layerand serum-free culture of human embryonic stem cells. Biol Reprod 2004; 70:837-845.

52 Beattie GM, Lopez AD, Bucay N, et al. Activin A maintains pluripotency of human embryonic stem cells in the absence of feeder layers. Stem Cells 2005; 23:489-495.

53 Prowse AB, McQuade LR, Bryant KJ, et al. A proteome analysis of conditioned media from human neonatal fibroblasts used in the maintenance of human embryonic stem cells. Proteomics 2005; 5:978-989.

54 Lim JW, Bodnar A. Proteome analysis of conditioned medium from mouse embryonic fibroblast feeder layers which support the growth of human embryonic stem cells. Proteomics 2002; 2:1187-1203

55 Levenstein ME, Ludwig TE, Xu RH, et al. Basic fibroblast growth factor support of human embryonic stem cell self-renewal. Stem Cells 2006; 24:568-574.

$56 \mathrm{Xu}$ RH, Peck RM, Li DS, et al. Basic FGF and suppression of BMP signaling sustain undifferentiated proliferation of human ES cells. Nat Methods 2005; 2:185-190.

57 Stojkovic P, Lako M, Przyborski S, et al. Human-serum matrix supports undifferentiated growth of human embryonic stem cells. Stem Cells 2005; 23:895-902.

58 Liu Y, Song Z, Zhao Y, et al. A novel chemical-defined medium with bFGF and N2B27 supplements supports undifferentiated growth in human embryonic stem cells. Biochem Biophys Res Commun 2006; 346:131-139.

59 Lu J, Hou R, Booth CJ, Yang SH, Snyder M. Defined culture conditions of human embryonic stem cells. Proc Natl Acad Sci USA 2006; 103:5688-5693.

60 Yao S, Chen S, Clark J, et al. Long-term self-renewal and directed differentiation of human embryonic stem cells in chemically defined conditions. Proc Natl Acad Sci USA 2006; 103:69076912.

61 Fletcher JM, Ferrier PM, Gardner JO, et al. Variations in humanized and defined culture conditions supporting derivation of new human embryonic stem cell lines. Cloning Stem Cells 2006; 8:319-334.

62 Draper JS, Smith K, Gokhale P, et al. Recurrent gain of chromosomes $17 \mathrm{q}$ and 12 in cultured human embryonic stem cells. Nat Biotechnol 2004; 22:53-54.

63 Gruen L, Grabel L. Concise review: scientific and ethical roadblocks to human embryonic stem cell therapy. Stem Cells 2006; 24:2162-2169. 